\title{
Helicobacter pylori treatment results in Slovenia in the period 2013-2015 as a part of European Registry on Helicobacter pylori Management
}

\author{
Bojan Tepes ${ }^{1}$, Marko Kastelic ${ }^{1}$, Miroslav Vujasinovic², Polona Lampic ${ }^{3}$, Maja Seruga ${ }^{4}$, \\ Natasa Brglez Jurecic ${ }^{5}$, Olga P. Nyssen ${ }^{6}$, Maria G. Donday ${ }^{6}$, Colm O’Morain ${ }^{7}$, \\ Francis Megraud ${ }^{8}$, Adrian G McNicholl ${ }^{6}$, Javier P. Gisbert ${ }^{6}$ \\ ${ }^{1}$ Abakus Medico, Diagnostic Center Rogaška, Rogaška Slatina, Slovenia \\ ${ }^{2}$ General Hospital Slovenj Gradec, Slovenj Gradec, Slovenia \\ ${ }^{3}$ Diagnostic Center Bled, Bled, Slovenia \\ ${ }^{4}$ General Hospital Murska Sobota, Murska Sobota, Slovenia \\ ${ }^{5}$ General Hospital Trbovlje, Trbovlje, Slovenia \\ ${ }^{6}$ Gastroenterology Unit, Hospital Universitario de La Princesa, Instituto de Investigación Sanitaria Princesa (IIS-IP) and \\ Centro de Investigación Biomédica en Red de Enfermedades Hepáticas y Digestivas (CIBEREHD), Madrid, Spain \\ ${ }^{7}$ Beacon Consultants Clinic, Dublin, Ireland \\ ${ }^{8}$ Laboratoire de Bactériologie-Enfants Hôpital Pellegrin 33076 Bordeaux cedex, France
}

Radiol Oncol 2018; 52(1): 1-6.

Received 11 September 2017

Accepted 5 November 2017

Correspondenece to: Prof. Bojan Tepeš M.D., Ph.D., FEBGH, AM DC Rogaška, Prvomajska 29 A, 3250 Rogaška Slatina, Slovenia. E mail: bojan.tepes@siol.net

Disclosure: No potential conflicts of interest were disclosed.

\begin{abstract}
Background. Helicobacter pylori (H. pylori) is the most common chronic bacterial infection in the world affecting over $50 \%$ of the world's population. H. pylori is a grade I carcinogen, responsible for the development of $89 \%$ of noncardia gastric cancers. In the present study we analyzed the data for $\mathrm{H}$. pylori eradication treatments in Slovenia.

Patients and methods. Slovenia is a part of the European Registry on Helicobacter pylori Management from the beginning. In seven medical institutions data for $\mathrm{H}$. pylori eradication treatments was collected for 1774 patients from April $16^{\text {th }} 2013$ to May 15 th 2016. For further modified intention to treat (mITT) analysis 1519 patients were eligible and for per protocol (PP) analysis 1346 patients.

Results. Patients' dropout was $11.4 \%$. Eradication rate for 7 day triple therapy with proton pump inhibitor (PPI) + Clarithromycin (C) + Amoxicillin (A) was $88.7 \%$ PP and $72.0 \% \mathrm{mlTT}$; for PPI + C + Metronidazole (M) $85.2 \%$ PP and $84.4 \%$ $\mathrm{mITT}$. Second line 14 day therapy PPI + A + Levofloxacin had $92.3 \%$ eradication rate PP and $87.1 \% \mathrm{mITT}$. Ten to fourteen day Bismuth quadruple therapy was the therapy in difficult to treat patients. At the end all patients that adhered to prescribed regimens were cured of their $H$. pylori infection.

Conclusions. High dropout rate deserves further analysis. Slovenia is still a country with $<15 \% \mathrm{H}$. pylori resistance to clarithromycin, triple therapy with PPI plus two antibiotics reaches PP eradication rate $>85 \%$, but mlTT eradication rates are suboptimal.
\end{abstract}

Key words: Helicobacter pylori; eradication treatment; European Registry on Helicobacter pylori management; Slovenian results

\section{Introduction}

Helicobacter pylori (H. pylori) is the cause of the most common chronic bacterial infection in the world, affecting over $50 \%$ of the world's popula- tion. ${ }^{1}$ Approximately $20 \%$ of infected patients will develop peptic ulcer disease, mucosa associated lymphoid tissue lymphoma or gastric cancer at some point in their lives. ${ }^{2}$ The WHO's International Agency for Research on Cancer classified H. pylori 
as a definite (group 1) carcinogen. ${ }^{3,4} \mathrm{H}$. pylori is the leading infectious cause of cancer worldwide and it was estimated that some 660000 new cancer cases globally were directly attributable to $H$. pylori infection in 2008, which accounts for 33\% of all infection-associated cancers, including $46 \%$ in developed regions and $29 \%$ in the developing regions. ${ }^{5}$

Several national guidelines have recommended eradication in all $H$. pylori-infected individuals to prevent the spread of infection and reduce the future burden of $H$. pylori-induced diseases, particularly gastric cancer. ${ }^{6,7}$ In the Kyoto consensus on $H$. pylori gastritis the recommendation is to treat all H. pylori infected patients independent of whether clinical manifestations are present. ${ }^{8}$

The basis of modern H. pylori therapy is a proton pump inhibitor (PPI) plus two / three antibiotics for 7-14 days. $^{2}$ The desired eradication rate is $>90 \%$, the acceptable eradication rate for first line therapy is $>80 \%{ }^{9}$

The treatment success depends on $H$. pylori's susceptibility to antibiotics and patient's compliance. $H$. pylori resistance rate to antibiotics as well as treatment results should be carefully recorded and those results should guide the therapy in a certain country or region. ${ }^{2}$ The European Registry on H. pylori management (Hp-EuReg) has been introduced in 2013 with the idea to collect treatment results, side effects, patients' compliance and antimicrobial susceptibility in different European countries. Thirty one countries and 280 recruiting investigators are included in the Hp-EuReg. So far, more than 15.000 patients have been included, and 12.270 patients have finished follow-up. ${ }^{10}$

We would like to present the Slovenian data collected in Hp-EuReg from April 16 2013 to May $15^{\text {th }} 2016$.

\section{Patients and methods}

\section{European Registry on $H$. pylori Management}

The present manuscript is an interim analysis using the Slovenian data of the "European Registry on H. pylori Management" (Hp-EuReg), an international multicenter prospective non-interventionist registry that will last over ten years, promoted by the European Helicobacter and Microbiota Study Group (www.helicobacter.org). The Scientific Committee of the project is comprised by Javier P. Gisbert (Principal Investigator), Francis Megraud, Colm O'Morain and Adrian G. McNicholl (Scientific Coordinator).

\section{Ethics}

Hp-EuReg protocol was approved by the Ethics Committee of the La Princesa Hospital (Madrid, Spain) that acted as reference IRB, and was prospectively registered at ClinicalTrials.gov under code NCT02328131.

\section{National coordinators}

A list of 30 European Countries has been selected. In each country a National Coordinator was elected based on its clinical and research activity. Slovenian National Coordinator is Bojan Tepes. The National Coordinators constitutes the monitoring and drafting committee of the registry in a certain country.

\section{Recruiter investigators}

The Recruiting Investigators are gastroenterologists attending an adult population with a gastroenterology outpatient clinic that assists $H$. pylori infected patients. Eradication confirmation tests have to be performed routinely. Patients are managed and registered following routine clinical practice. Slovenian recruiting investigators are authors BT, MK, MV, PL, MS and NBJ.

\section{Electronic Case Report Form (e-CRF)}

Study data were collected and managed using REDCap electronic data capture tools hosted at Asociación Española de Gastroenterología (AEG; www.aegastro.es). ${ }^{11}$ AEG is a non-profit Scientific and Medical Society focused on Gastroenterology, and it provided this service free of charge, with the sole aim of promoting independent investigator driven research. REDCap (Research Electronic Data Capture) is a secure, web-based application designed to support data capture for research studies, providing (1) an intuitive interface for validated data entry; (2) audit trails for tracking data manipulation and export procedures; (3) automated export procedures for seamless data downloads to common statistical packages; and (4) procedures for importing data from external sources.

\section{Variables and outcomes}

The e-CRF includes 290 variables including demographics, history and comorbidity, data on infection and diagnosis, previous eradication attempts, current treatment, compliance, adverse events and 
efficacy. All patient data was anonymized. Main outcome is confirmed eradication at least 4 weeks after treatment.

Per protocol (PP) analysis include all patients who finished follow-up and took at least $90 \%$ of the treatment drugs, as defined in the approved protocol. As the registry is ongoing, a pure Intention to treat (ITT) analysis cannot be provided. A modified ITT (mITT) was designed trying to reach the closest result to those obtained in clinical practice. This mITT includes for analyses all patients whose outcome has been registered by their doctors (eradication success, failure or lost to follow up), plus those that although their result has not been registered were treated more than a year prior to analysis. Patients classified as failure, lost or without registered outcome will be considered treatment failure in the mITT analysis.

\section{Statistical analyses}

Continuous variables are presented as the arithmetic mean and SDs. Qualitative variables are presented as proportions and $95 \%$ confidence intervals (CIs). The statistical analyses of the results were carried out using the $\chi 2$-test. The $95 \% \mathrm{CIs}$ were calculated by normal approximation. Oneand two-sided tests were used for the analyses and the P-value cut off for significance was set to less than 0.05 . The analyses were carried out using IBM SPSS Statistics 22.0.0.

\section{Results}

Data was collected in seven medical institutions in Slovenia from April 16 ${ }^{\text {th }} 2013$ to May $15^{\text {th }} 2016$ for 1774 patients (Table 1).

Two hundred and fifty-five $(14.4 \%)$ patients were excluded from the analysis because they either had incomplete/invalid data (e.g.: missing age, gender, compliance data etc.) or because they were treated within the last year of this analysis and the outcome of their treatment was not yet registered.

All the remaining 1519 patients were eligible for further analysis in the modified intention to treat (mITT) group; 918 (60.4\%) were female patients and $601(39.6 \%$ ) were male patients (Table 2; $\mathrm{p}=0.00$ ).

Out of those, 1346 patients had their outcome registered and were eligible for analysis in the per protocol (PP) group.

There were 173 patients $(11.4 \%)$ who did not have their outcome recorded and were treated more than a year prior to this analysis. We consider
TABLE 1. Medical institutions participating in the Slovenian part of EU-HpReg

\begin{tabular}{lcccccc}
\hline Institution & \multicolumn{2}{c}{ Number } & \multicolumn{2}{c}{$\begin{array}{c}\text { Excluded from analysis } \\
\text { (\% of hosp. data) }\end{array}$} & \multicolumn{2}{c}{ Dropout (\%) } \\
\hline AM DC Rogaska & 805 & $(45.4 \%)$ & 146 & $(18.1 \%)$ & 16 & $(2.4 \%)$ \\
SB Slovenj Gradec & 464 & $(26.2 \%)$ & 8 & $(1.7 \%)$ & 81 & $(17.8 \%)$ \\
DC Bled & 287 & $(16.2 \%)$ & 0 & $(0 \%)$ & 60 & $(20.9 \%)$ \\
SB Murska Sobota & 73 & $(4.1 \%)$ & 10 & $(13.7 \%)$ & 0 & $(0 \%)$ \\
SB Trbovlje & 68 & $(3.8 \%)$ & 34 & $(50 \%)$ & 1 & $(2.9 \%)$ \\
UKC Ljubljana & 66 & $(3.7 \%)$ & 50 & $(75.8 \%)$ & 14 & $(87.5 \%)$ \\
MC Heliks & 11 & $(0.6 \%)$ & 7 & $(63.6 \%)$ & 1 & $(25 \%)$ \\
Total & 1774 & $(100.0 \%)$ & 255 & $(14.4 \%)$ & 173 & $(11.4 \%)$ \\
\hline
\end{tabular}

' Excluded from analysis because of incomplete/invalid data or because the visit was within last year and there is no follow up data yet; ${ }^{2}$ Patients whose visit was more than a year ago and who had no follow up (dropout $=$ modified intention to treat [mlTT] - per protocol [PP])

TABLE 2. Demographic data for modified intention to treat (mITT) patient group

\begin{tabular}{lccccc}
\hline Gender & N (percent) & $\begin{array}{c}\text { Minimum } \\
\text { Age }\end{array}$ & $\begin{array}{c}\text { Maximum } \\
\text { Age }\end{array}$ & $\begin{array}{c}\text { Mean } \\
\text { Age }\end{array}$ & $\begin{array}{c}\text { Std. } \\
\text { Deviation }\end{array}$ \\
\hline Female & $918(60.4 \%)$ & 18 & 91 & 52.3 & 15.14 \\
Male & $601(39.6 \%)$ & 18 & 88 & 53.3 & 14.76 \\
Total & 1519 & 18 & 91 & 52.7 & 15.0 \\
\hline
\end{tabular}

that group a drop out patients group. We do not know if these patients took their therapy or whether they had the UBT done in the primary medical care and because of that did not return to their gastroenterologist, which can be a realistic option.

Only in 56 patients who took part in a RCT, primary $H$. pylori resistance to antibiotics was recorded (Table 3). The highest resistance rate was for metronidazole (M; $28.6 \%)$, resistance rate to clarithromycin (C) was $14.3 \%$ and to amoxicillin (A) was 3.6\%. All the other antimicrobial susceptibility tests have been performed in treatment failure patients. The percentage of resistance to different antibiotics rose with the number of treatment attempts.

Ten different 7-14 days triple combinations were used in 1305 patients as a first line treatment. The majority of patients (1.154) were treated accordingly to our therapeutic guidelines (Table 4) with 7 day triple therapies: PPI clarithromycin / amoxicillin / metronidazole. The eradication rate for PPI + Clarithromycin + Amoxicillin was $72.0 \%$ for the mITT group vs. $88.1 \%$ for the PP group. The eradication rate in 7 day PPI + Clarithromycin + Metronidazole was $84.4 \%$ for the mITT group vs. $85.2 \%$ for the PP group. No significant differences were found regarding the type of PPI used.

Six different triple 7-14 days treatments were used in 176 patients whose $H$. pylori was not eradicated with the first line treatment (Table 5). The 
TABLE 3. Helicobacter pylori antibiotic resistance

\begin{tabular}{|c|c|c|c|c|c|c|c|c|}
\hline \multirow{2}{*}{$\begin{array}{l}\text { Antibiotic } \\
\text { No resistance }\end{array}$} & \multicolumn{2}{|c|}{$\begin{array}{l}\text { First treatment } \\
(95 \% \text { C.I. })\end{array}$} & \multicolumn{2}{|c|}{$\begin{array}{l}\text { Second treatment } \\
(95 \% \text { C.I. })\end{array}$} & \multicolumn{2}{|c|}{$\begin{array}{l}\text { Third treatment } \\
(95 \% \text { C.I. })\end{array}$} & \multirow{2}{*}{$\begin{array}{c}\begin{array}{c}\text { Fourth } \\
\text { treatment }\end{array} \\
0 \%\end{array}$} & \multirow{2}{*}{$\begin{array}{c}\begin{array}{c}\text { Fifth } \\
\text { treatment }\end{array} \\
0\end{array}$} \\
\hline & $62.5 \%$ & (50.0\%-75.4\%) & $6.1 \%$ & $(0 \%-15.6 \%)$ & $14.3 \%$ & $(0 \%-36.4 \%)$ & & \\
\hline Nitroimidazole & $28.6 \%$ & (16.7\%-40.8\%) & $45.5 \%$ & (28.1\%-63.0\%) & $57.1 \%$ & (30.0\%-83.3\%) & $100 \%$ & $100 \%$ \\
\hline Clarithromycin & $14.3 \%$ & (5.7\%-24.2\%) & $87.9 \%$ & (75.7\%-97.3\%) & $85.7 \%$ & (63.6\%-100\%) & $100 \%$ & $100 \%$ \\
\hline Amoxicillin & $3.6 \%$ & (0\%-9.3\%) & $0 \%$ & & $0 \%$ & & $0 \%$ & 0 \\
\hline Quinolone & $0 \%$ & & $6.1 \%$ & $(0 \%-15.6 \%)$ & $7.1 \%$ & $(0 \%-23.5 \%)$ & $50 \%$ & 0 \\
\hline Tetracycline & $0 \%$ & & $3 \%$ & $(0 \%-100 \%)$ & $7.1 \%$ & $(0 \%-23.5 \%)$ & $0 \%$ & 0 \\
\hline Total & $N=56$ & & & $N=33$ & & $N=14$ & $N=2$ & $N=1$ \\
\hline
\end{tabular}

C. I. = confidence interval

majority of them used 14 days PPI $40 \mathrm{mg}$ bid, Amoxicillin (A) $1000 \mathrm{mg}$ bid, and Levofloxacin (L) $500 \mathrm{mg}$ oid with $92.3 \%$ eradication rate PP and $87.1 \%$ eradication rate mITT. This treatment is according to our guidelines the recommended one for second line. Dropout rate for the second treatment attempt was $6.3 \%$.

Bismuth is not available in Slovenia and those that need third or fourth line treatment regimen should buy it in Germany or in any other country in Europe where it is available. At the moment this treatment is not reimbursed (Tables 6,7). Dropout rate after third line therapy was $18.2 \%$. Seven patients were treated with fourth line treatment regimen (Table 7), one was treated with fifth, and one with sixth line treatment. No drop out has been recorded in the group with four or more treatment attempts.

At the end all patients that start their treatment and comply with the treatment regimens were cured of their $H$. pylori infection.

\section{Discussion}

High dropout rate - $11.4 \%$ - in the Slovenian HpEuReg data is the first important message. This per- centage is lower than in the EU Hp-EuReg (13\%). ${ }^{10}$ Some logistic reasons can influence this high dropout rate. All 66 patients (3.73\% of all patients) from University Medical Centre did not come back to their gastroenterologist. They were most probably controlled by their general practitioner, but we are not aware of their UBT results. And many more dropout patients from other medical centers could be treated in the same way. Real patients' compliance was never an issue, also in our previous reports / studies. ${ }^{12,13}$

H. pylori resistance to clarithromycin is still low in Slovenia. It was $10.5 \%$ in a recently published study and $25.9 \%$ for metronidazole. ${ }^{12}$ In our HpEuReg data primary resistance to clarithromycin was recorded in a small subgroup of patients and was $14.3 \%$ and $28.6 \%$ for metronidazole. That can explain the still relatively good eradication results for 7 day triple therapy (TT). The PP eradication rate for PPI A C was 88.7\% (72.0\% mITT) and 85.2\% PP for PPI C M (84.4\% mITT). This is an acceptable eradication rate in PP analysis, but still not satisfactory, because it did not reach $>90 \%$ eradication rate. In some other parts of the world due to the increasing incidence of $H$. pylori resistance to clarithromycin, the cure rates of 7 day triple therapy (TT) have decreased to less than $80 \%$, which is

TABLE 4. First line treatment results for treatment regimens with more than 15 patients

\begin{tabular}{|c|c|c|c|c|c|c|}
\hline \multirow{3}{*}{$\begin{array}{l}\text { Treatment } \\
\text { Clarythromycin, amoxicillin, PPI, } 7 \text { days }\end{array}$} & \multicolumn{3}{|c|}{ mITt } & \multicolumn{3}{|c|}{ PP } \\
\hline & \multirow{2}{*}{$\frac{N}{664}$} & \multicolumn{2}{|c|}{ Success \% (95\% C.I.) } & \multirow{2}{*}{$\frac{\mathbf{N}}{538}$} & \multicolumn{2}{|c|}{ Success \% (95\% C.I.) } \\
\hline & & $72.0 \%$ & $(68.6 \%-75.4 \%)$ & & $88.7 \%$ & $(85.9 \%-91.3 \%)$ \\
\hline Clarythromycin, metronidazole, PPI,7 days & 486 & $84.4 \%$ & $(81.2 \%-87.5 \%)$ & 481 & $85.2 \%$ & $(82.0 \%-88.4 \%)$ \\
\hline Clarythromycin, amoxicillin, PPI, 10 days & 38 & $55.3 \%$ & (39.1\%-71.1\%) & 27 & $77.8 \%$ & (60.7\%-92.9\%) \\
\hline Clarythro., amoxicillin., metro., PPI, 7 days & 17 & $88.2 \%$ & $(70.6 \%-100 \%)$ & 15 & $93.3 \%$ & $(77.8 \%-100 \%)$ \\
\hline
\end{tabular}

metro. $=$ metronidazole $; \mathrm{mITT}=$ modified intention to treat; $\mathrm{PP}=$ per protocol; $\mathrm{PPI}=$ proton pump inhibitor 
TABLE 5. Second line treatment results for treatment regimens with more than 15 patients

\begin{tabular}{|c|c|c|c|c|c|c|}
\hline \multirow{3}{*}{$\begin{array}{l}\text { Treatment } \\
\text { Amoxicillin, Levofloxacin, PPI, } 14 \text { days }\end{array}$} & \multicolumn{3}{|c|}{$\mathrm{mlTt}$} & \multicolumn{3}{|c|}{ PP } \\
\hline & \multirow{2}{*}{$\begin{array}{c}\mathbf{N} \\
70\end{array}$} & \multicolumn{2}{|c|}{ Success $\%$ ( $95 \%$ C.I.) } & \multirow{2}{*}{$\begin{array}{l}N \\
65\end{array}$} & \multicolumn{2}{|c|}{ Success \% ( $95 \%$ C.I.) } \\
\hline & & $87.1 \%$ & $(78.8 \%-94.6 \%)$ & & $92.3 \%$ & $(85.3 \%-98.3 \%)$ \\
\hline Amoxicillin, Levofloxacin, PPI, 10 days & 24 & $91.7 \%$ & $(78.6 \%-100 \%)$ & 23 & $95.7 \%$ & $(85.7 \%-100 \%)$ \\
\hline Amoxicillin, Metronidazole, PPI, 7 days & 18 & $44.4 \%$ & $(21.1 \%-68.4 \%)$ & 17 & $47.1 \%$ & $(23.1 \%-72.2 \%)$ \\
\hline Amoxicillin, Metronidazole, PPI, 10 days & 16 & $87,5 \%$ & $(68.4 \%-100 \%)$ & 15 & $93.3 \%$ & (77.8\%-100\%) \\
\hline
\end{tabular}

C. I. = confidence interval; $\mathrm{mITT}=$ modified intention to treat; $\mathrm{PP}=$ per protocol; $\mathrm{PPI}=$ proton pump inhibitor

considered to be unacceptably low (14). Maastricht IV suggest not to use 7 day triple therapy in countries with $H$. pylori resistance to clarithromycin over $15 \%$. Our H. pylori resistance rate to clarithromycin is still under this value, but we must continue to audit eradication rates with triple therapies in Slovenia as well as continuously measure primary H. pylori resistance rate to clarithromycin and other antibiotics.

Ten different 7-14 days triple combinations were used as a first line therapy. In our last $H$. pylori treatment guidelines ${ }^{7}$ only two TT (PPI A C and PPI C M) were recommended. This shows us that real clinical practice in Slovenia is not ideal, which was also recognized in some other countries shown in Hp-EuReg data. ${ }^{10}$

We did not show any benefits of esomeprazole over other PPIs in the eradication rates as was shown in some other studies. ${ }^{10,15}$ Because Caucasians have higher prevalence of high metabolizers (56\%-81\%) compared to Asians, higher doses of esomeprazole or rabeprazole are recommended, as they can control gastric $\mathrm{pH}$ adequately and allow better antibiotic efficacy. ${ }^{16,17}$

When H. pylori infection is found, it is very important to control the eradication rate and prescribe second or even further treatments to cure the infection. ${ }^{2}$ In our data, dropout rate for second line therapy is still too high (6.3\%). Results in HpEuReg are even worse with only $27 \%$ of first line eradication failures being retreated. ${ }^{10}$ In Maastricht IV quadruple bismuth therapy is recommended as the second line treatment in low clarithromycin resistant regions. ${ }^{2}$ The fact is that this therapy is not available in Slovenia at this moment. That is why the majority of second line treatments were 14 days PPI A L. In the National recommendation ${ }^{7}$ only 14 day therapy should be used as a second line treatment. But in real practice, gastroenterologists use therapies from 7-14 days. This variations need to be corrected, because longer duration of second line therapies mean also better cure rates., ${ }^{2,18}$ Our eradication rate for PPI A L is $92.3 \%$ in PP group and $87.1 \%$ in mITT group, which is a satisfactory result. We know that this is due to low $H$. pylori resistance to quinolones in Slovenia (3.1-4.4\%). ${ }^{19,20}$

Other possible second line treatment could also be sequential or non-bismuth concomitant therapy with PPI and all three antibiotics (C, M, E). This therapy has been proven to be very effective (>90\% by ITT) in Slovenia ${ }^{10}$ but is not used at the moment in clinical practice. These therapies can be effective also in the regions with $H$. pylori resistance to clarithromycin $>15 \% .{ }^{21-23}$ Only if dual resistance to clarithromycin and metronidazole is $>15 \%$, the eradication rate of non-bismuth quadruple therapies will be impaired and bismuth quadruple therapies should be used. ${ }^{24}$

In the third and fourth line treatment PPI A L was used in some patients not treated with this regimen before, as well as bismuth quadruple therapies. Some patients were treated with 14 day therapies, but not all, which should also be corrected.

In Maastricht recommendations culture and antibiotic susceptibility should be done after two unsuccessful therapeutic attempts ${ }^{2}$, but we seldom use this approach. The reason for this is non-reimbursement for culture by our National health fund.

One patient has been treated for the fifth time and one for the sixth time, both successfully. So finally all patients who were compliant with the prescribed therapeutic regimens were eradicated of $H$. pylori infection in the end, which is similar to our and international previously published data. ${ }^{13,25}$

\section{Conclusions}

Hp-EuReg is a very important clinical registry which helps us audit real clinical practice in the field of $H$. pylori eradication as well as collect eradication rates for different first, second line 
and beyond treatments. From the analysis of our Slovenian data we can figure out some clinically important conclusions:

Seven days PPI A/M /C results has unacceptable low mITT eradication rates. Treatment duration should be prolonged to 14 days.

Dropout rate is too high. We must provide all general practitioners with the possibility to use urea breath test or monoclonal stool antibody test in all patients with $H$. pylori eradication therapies. No patients should be without confirmation of eradication success.

Treatment failures of the first line regimen should be retreated according to National guidelines, that is with 14 day PPI A L regimen

Primary $H$. pylori resistance to antibiotics and treatment results should be continuously monitored

\section{Acknowledgements}

We want to thank the data monitors of the study, Mercedes Ramas, Lorena Lee and Irene Barbado for their large efforts to ensure the validity of the data and quality of their work. We want to thank Pau Alarcón who performed advanced data managing and programming for this project. We want to thank the Spanish Association of Gastroenterology (AEG) and the Scientific Director of AEG-REDCap (Adrian G. McNicholl) for providing the e-CRF service free of charge.

\section{References}

1. Hunt RH, Xiao SD, Megraud F, Leon-Barua R, Bazzoli F, van der Merwe $S$, et al. World Gastroenterology Organization. Helicobacter pylori in developing countries. World Gastroenterology Organisation Global Guideline. J Gastrointestin Liver Dis 2011; 20: 299-304. doi: 10.1097/ MCG.0b013e31820fb8f6

2. Malfertheiner P, Megraud F, O'Morain CA, Atherton J, Axon ATR, Bazzoli $F$, et al. Management of Helicobacter pylori infection - the Maastricht IV/Florence Consensus Report. Gut 2012; 61: 646-64. doi: 10.1136/ gutjnl-2012-302084

3. International Agency for Research on Cancer. Infection with Helicobacte pylori. IARC Monogr Eval Carcinog Risks Hum 1994; 61: 177-240.

4. IARC Helicobacter pylori Working Group (2014). Helicobacter pylor Eradication as a Strategy for Preventing Gastric Cancer. Lyon, France: International Agency for Research on Cancer IARC Working Group Reports 2014; 8: 1-181.

5. de Martel C, Ferlay J, Franceschi S, Vignat J, Bray F, Forman D, Plummer M. Global burden of cancers attributable to infections in 2008: a review and synthetic analysis. Lancet Oncol 2012; 13: 607-15. doi: 10.1016/S14702045(12)70137-7

6. Asaka M, Kato M, Takahashi S, Fukuda Y, Sugiyama T, Ota $H$, et al. Japanese Society for Helicobacter Research. Guidelines for the management of Helicobacter pylori infection in Japan: 2009 revised edition. Helicobacter 2010; 15: 1-20. doi: 10.1111/j.1523-5378.2009.00738.x
7. Tepeš B, Štabuc B. [Slovenian Society for Gastroenterology and Hepatology Guidelines on the management of Helicobacter pylori infection]. Guidelines on the management of Helicobacter pylori infection. [Slovenian]. Zdrav Vestn 2011; 80: 647-56.

8. Sugano K, Tack J, Kuipers EJ, Graham DY, El-Omar EM, Miura S, et al. Kyoto global consensus report on Helicobacter pylori gastritis. Gut 2015; 64: 1353 67. doi: 10.1136/gutjnl-2015-309252

9. Graham DY, Dorea MP. Helicobacter pylori therapy: a paradigm shift. Expert Rev Anti-infe 2016; 14: 577-85. doi: 10.1080/14787210.2016.1178065

10. McNicholl AG, Gasbarrini A, Tepeš B, Bordin DS, Lerang F, Leja M, et al. PanEuropean registry on $\mathrm{H}$. pylori management (HP-EUREG). Gastroenterology 2016; 150: S875-76. doi: 10.1016/S0016-5085(16)32952-3

11. Harris PA, Taylor R, Thielke R, Payne J, Gonzalez N, Conde JG. Research electronic data capture (REDCap) - A metadata-driven methodology and workflow process for providing translational research informatics support. $J$ Biomed Inform 2009; 42: 377-81. doi: 10.1016/j.jbi.2008.08.010

12. Tepeš $B$, Vujasinović $M$, Šeruga $M$, Stefanovič $M$, Forte $A$, Jeverica $S$. Randomized clinical trial comparing 10-day sequential, 7-day concomitant and 7-day standard triple therapies for Helicobacter pylori eradication. Eur J Gastroenterol Hepatol 2016; 28: 676-83. doi: 10.1097/ MEG.0000000000000590

13. Tepeš B, Zvezdana O. [Cumulative Helicobacter pylori eradication rates in Slovenia in the year 2008]. [Slovenian]. Zdrav Vestn 2010; 79: 19-24.

14. Graham DY, Fischbach L. Helicobacter pylori treatment in the era of increasing antibiotic resistance. Gut 2010; 59: 1143-53. doi: 10.1136/ gut.2009.192757

15. McNicholl AG, Linares PM, Nyssen OP, Calvet X, Gisbert JP. Meta-analysis: esomeprazole or rabeprazole vs. first-generation pump inhibitors in the treatment of Helicobacter pylori infection. Aliment Pharmacol Ther 2012; 36: 414-25. doi: 10.1111/j.1365-2036.2012.05211.x

16. Serrano D, Torrado S, Torrado-Santiago S, Gisbert J P. The influence of CYP2C19 genetic polymorphism on the pharmacokinetics/- pharmacodynamics of proton pump inhibitor-containing Helicobacter pylori treatments. Curr Drug Metab 2012; 13: 1303-12. doi: 10.2174/138920012803341393

17. Tang HL, Li Y, Hu YF, Xie HG, Zhai SD. Effects of CYP2C19 loss-of-function variants on the eradication of $H$. pylori infection in patients treated with proton pump inhibitor-based triple therapy regimens: a meta-analysis of randomized clinical trials. PLoS One 2013; 8: e62162. doi: 10.1371/journal. pone. 0066730

18. Tai WC, Lee $\mathrm{CH}$, Chiou SS, Kuo $\mathrm{CM}$, Kuo $\mathrm{CH}$, et al. The clinical and bacteriological factors for optimal levofloxacin-containing triple therapy in second-line Helicobacter pylori eradication. PLOS One 2014; 9: e105822. doi: 10.1371/journal.pone.0105822

19. Jeverica S, Tepeš B, Ihan A, Skvarč M. [Primary resistance of Helicobacter pylori]. [Slovenian]. Zdrav Vestn 2010; 79: 25-30

20. Megraud F, Coenen S, Versporten A, Kist M, Lopez-Brea M, Hirschl AM, et al. Helicobacter pylori resistance to antibiotics in Europe and its relationship to antibiotic consumption. Gut 2013; 62: 34-42. doi: 10.1136/ gutjnl-2012-302254

21. Molina-Infante J, Lucendo AJ, Angueira T, Rodriguez-Tellez M, Perez-Aisa A et al. Optimised empiric triple and concomitant therapy for Helicobacter pylori eradication in clinical practice: the OPTRICON study. Aliment Pharmacol Ther 2015; 41: 581-9. doi: 10.1111/apt.13069

22. McNicholl AG, Marin AC, Molina-Infante J, Castro M, Barrio J, Ducons, et al. Randomised clinical trial comparing sequential and concomitant therapies for Helicobacter pylori eradication in routine clinical practice. Gut 2014; 63: 244-9. doi: 10.1136/gutjnl-2013-304820

23. Molina-Infante J, Gisbert JP. Optimizing clarithromycin-containing therapy for Helicobacter pylori in the era of antibiotic resistance. World J Gastroenterol 2014; 20: 10338-47. doi: 10.3748/wjg.v20.i30.10338

24. Graham DY, Lee YC, Wu MS. Rational Helicobacter pylori therapy: evidencebased medicine rather than medicine-based evidence. Clin Gastroenterol Hepatol 2014; 12: 177-86. doi: 10.1016/j.cgh.2013.05.028

25. Rokkas T, Sechopoulos P, Robotis I, MargantinisG, Pistiolas D. Cumulative $H$. pylori eradication rates in clinical practice by adopting first and second line regimens proposed by Maastricht III consensus and a third line empirical regimen. Am J Gastroenterol 2009; 104: 21-5. doi: 10.1038/ajg.2008.87 